\title{
The systemic environment: at the interface of aging and adult neurogenesis
}

\author{
Lucas K. Smith ${ }^{1,2,3}$ • Charles W. White III ${ }^{1,2,4}$ - Saul A. Villeda ${ }^{1,2,3,4}$
}

Received: 6 June 2017 / Accepted: 9 October 2017 / Published online: 9 November 2017

(C) The Author(s) 2017. This article is an open access publication

\begin{abstract}
Aging results in impaired neurogenesis in the two neurogenic niches of the adult mammalian brain, the dentate gyrus of the hippocampus and the subventricular zone of the lateral ventricle. While significant work has characterized intrinsic cellular changes that contribute to this decline, it is increasingly apparent that the systemic environment also represents a critical driver of brain aging. Indeed, emerging studies utilizing the model of heterochronic parabiosis have revealed that immune-related molecular and cellular changes in the aging systemic environment negatively regulate adult neurogenesis. Interestingly, these studies have also demonstrated that age-related decline in neurogenesis can be ameliorated by exposure to the young systemic environment. While this burgeoning field of research is increasingly garnering interest, as yet, the precise mechanisms driving either the proaging effects of aged blood or the rejuvenating effects of young blood remain to be thoroughly defined. Here, we
\end{abstract}

Saul A. Villeda

saul.villeda@ucsf.edu

Lucas K. Smith

lucas.smith@ucsf.edu

Charles W. White, III

charles.white@ucsf.edu

1 Department of Anatomy, University of California, San Francisco, San Francisco, CA 94143, USA

2 The Eli and Edythe Broad Center of Regeneration Medicine and Stem Cell Research, University of California San Francisco, San Francisco, CA 94143, USA

3 Biomedical Sciences Graduate Program, University of California San Francisco, San Francisco, CA 94143, USA

4 Developmental and Stem Cell Biology Graduate Program, University of California San Francisco, San Francisco, CA 94143, USA review how age-related changes in blood, blood-borne factors, and peripheral immune cells contribute to the age-related decline in adult neurogenesis in the mammalian brain, and posit both direct neural stem cell and indirect neurogenic nichemediated mechanisms.

Keywords Aging $\cdot$ Rejuvenation · Adult neurogenesis · Blood $\cdot$ Immune cells

\section{Introduction}

Aging is a global process, resulting in disrupted homeostatic function and a loss of neurogenic capacity in the mammalian brain. Systemic interventions have recently been shown to regulate these effects in a variety of tissues, including the brain. Here, we will review the growing body of work that posits the systemic environment as a key regulator of the decline in neurogenesis observed in the aging mammalian brain, and will explore potential mechanisms by which the pro-aging and rejuvenating effects of the systemic milieu are mediated.

Traditionally, the adult mammalian brain was thought to be devoid of neural stem cell (NSC) activity, reliant largely on gene expression changes and structural reorganization for plasticity. In 1965, however, Altman and Das (1965) first observed evidence of newborn neurons in the adult rat hippocampus and, later, subventricular zone (SVZ). While controversial at the time, much work has since revealed that neurogenesis occurs throughout life in all vertebrate classes (Barker et al. 2011), including fish (Zupanc and Sirbulescu 2013), birds (Nottebohm 2004), reptiles (Chapouton et al. 2007) and in mammals ranging from rodents to humans (Amrein et al. 2011; Bond et al. 2015). In the adult mammalian hippocampus, neurogenesis occurs in a distinct region known as the dentate gyrus (DG). Specifically, NSCs involved 
in this process reside in the subgranular zone (SGZ), a thin, highly vascularized, layer of cells between the hilus and the densely populated granule cell layer. NSCs first enter a proliferative neuroblast state, replicating for a short time before beginning to migrate into the granule cell layer where they extend neuronal processes, taking on an immature neuronal identity after 2 weeks. Subsequently, mature differentiated neurons functionally integrate into the hippocampal circuit projecting to the CA3 after 4 weeks. In addition to the hippocampus, adult neurogenesis is observed in the SVZ of the mammalian brain. The NSC population of the SVZ resides along the walls of the lateral ventricles, in close proximity to the vasculature and cerebrospinal fluid (CSF). During adult neurogenesis, these cells adopt a proliferative neuroblast state and enter the rostral migratory stream, a path extending from the SVZ to the olfactory bulb. Upon reaching the olfactory bulb, these cells adopt either a granule or periglomerular cell fate, and migrate into the granule cell layer or glomerular layer of the olfactory bulb, respectively. In the years since its discovery, the physiological significance of adult neurogenesis has been further elucidated, with implications for cognitive function. Indeed, in the hippocampus, ablation of neurogenesis in mice has been shown to impair spatial learning and memory, pattern separation, and mood regulation (Zhang et al. 2008; Clelland et al. 2009; Deng et al. 2009; Christian et al. 2014; Tsai et al. 2015), while loss of SVZ neurogenesis leads to impaired odor discrimination (Valley et al. 2009; Sahay et al. 2011).

Neurogenesis has been shown to decrease in the hippocampus and SVZ of the adult mammalian brain as a function of age (Bizon et al. 2004; Enwere 2004; Amrein et al. 2011). Importantly, aging results in a structural remodeling of the DG and SVZ neurogenic niches, characterized by astrogliosis, microgliosis, and vascular remodeling, all of which may contribute to the decline in neurogenesis. Recently, much work has been carried out to understand how combinations of agerelated changes in both the niche and intrinsic cellular processes play a role in this loss of neurogenesis. In the hippocampus, a significant increase in the ratio of quiescent to active NSCs accompanies age, and suggests that a loss of NSC activity rather than number plays a role in cognitive decline (Lugert et al. 2010). At the neurogenic niche level, several age-related changes have been linked to changes in neurogenesis, including loss of fibroblast growth factor-2 and vascular endothelial growth factor signaling (Shetty et al. 2005), decreased Wnt signaling (Seib et al. 2013), and increases in bone morphogenic protein (BMP) signaling (Yousef et al. 2015a). In the olfactory bulb, age-dependent loss of function has been shown to result from a marked reduction in the capacity of cells in the niche to undergo neurogenesis in response to epidermal growth factor signaling (Enwere 2004). Furthermore, an age-dependent increase in $\mathrm{p} 16^{\mathrm{INK} 4 \mathrm{a}}$, an intrinsic factor commonly associated with senescence, within the progenitor population has also been shown to specifically impair neurogenesis within the SVZ (Molofsky et al. 2006). At a functional level, the role of the age-related decline in neurogenesis in mediating the age-related impairments in cognitive function remains controversial (Drapeau et al. 2003; Merrill et al. 2003; Seib et al. 2013). Notwithstanding, interventions that boost adult neurogenesis are consistently accompanied by cognitive enhancements in aged mice (Kempermann et al. 2002; van Praag et al. 2005; Kodali et al. 2015), raising the prospect that identifying regulators of age-related decline in neurogenic capacity could reveal potential targets for cognitive improvement at old age.

\section{Systemic environment: regulator of adult neurogenesis with age}

While cell-intrinsic and niche-related factors are important in the age-related decline in neurogenesis, it is increasingly appreciated that the systemic environment also plays a role in regulating stem cell function during aging. Studies using the model of heterochronic parabiosis, in which the circulatory systems of a young and aged mouse are surgically joined, have allowed for a deepened understanding of systemic regulation and coordination of aging in multiple tissues (reviewed in Castellano et al. 2015). To date, researchers have revealed that exposure to old blood inhibits regeneration in muscle (Conboy et al. 2005; Brack et al. 2007; Rebo et al. 2016) and liver (Conboy et al. 2005), while young blood exerts rejuvenating effects on bone repair (Baht et al. 2015), $\beta$-cell proliferation (Salpeter et al. 2013), cardiac hypertrophy (Loffredo et al. 2013), muscle (Conboy et al. 2005; Brack et al. 2007; Sinha et al. 2014), and liver (Conboy et al. 2005). Importantly, both the pro-aging effects of old blood and rejuvenating effects of young blood extend to the CNS (Villeda et al. 2011, 2014; Ruckh et al. 2012; Baruch et al. 2014; Katsimpardi et al. 2014; Rebo et al. 2016; Castellano et al. 2017).

Given the close proximity of adult NSCs to the vasculature of the brain (Fuentealba et al. 2012), it is perhaps unsurprising that adult NSCs are highly sensitive to exposure to old and young blood. Indeed, exposure to old blood in the model of parabiosis, leads to decreased proliferation in both the DG and SVZ (Villeda et al. 2011; Katsimpardi et al. 2014). Furthermore, young heterochronic parabionts exhibited a decrease in the numbers of neural progenitor cells (NPCs) in the DG and SVZ, accompanied by a decrease in differentiated mature neurons (Villeda et al. 2011; Katsimpardi et al. 2014). Conversely, young blood leads to increased NPC proliferation and neuronal differentiation and survival in both the DG and SVZ of aged heterochronic parabionts (Villeda et al. 2011; Katsimpardi et al. 2014). Interestingly, exposure to young blood appears to drive stable rejuvenating changes in 
aged NSCs, as SVZ NSCs isolated from old heterochronic parabionts exhibit increased proliferation and increased neurogenesis when cultured in vitro, compared to NSCs isolated from old isochronic controls (Katsimpardi et al. 2014). Together, these studies indicate that neurogenesis is regulated by blood in an age-dependent manner through effects on NSC function.

Recently, the anti-neurogenic effects of aged blood were further corroborated in an independent model of heterochronic blood exchange, in which the circulatory system of young and old mice are connected via a jugular venous catheter, and blood repeatedly exchanged over the course of $24 \mathrm{~h}$ (Rebo et al. 2016). Short-term exposure to old blood in this model led to a decrease in proliferating NPCs in the DG of young mice (Rebo et al. 2016). Interestingly, short-term exposure to young blood in this model did not recapitulate the rejuvenating effects observed in old heterochronic parabioints (Villeda et al. 2011; Katsimpardi et al. 2014), where young blood exposure is more long-term. These findings suggest that the rejuvenating effects of young blood on neurogenesis may only be apparent after prolonged exposure.

\section{Blood plasma: loss of pro-youthful and accumulation of pro-aging soluble factors}

Given the exciting rejuvenating effects observed in the model of heterochronic parabiosis, there is much interest in identifying blood-borne soluble factors that might underlie the proyouthful effects of young blood. While intravenous (I.V.) administration of young adult blood plasma recapitulates aspects of heterochronic parabiosis (Villeda et al. 2014), its effects on neurogenesis have not yet been investigated. Despite this, GDF11, a TGF- $\beta$ superfamily member, has emerged as a potential pro-youthful factor that boosts SVZ neurogenesis in old mice (Katsimpardi et al. 2014). GDF-11 was originally shown to decline in the blood of aged mice, and to rejuvenate aging phenotypes in heart (Loffredo et al. 2013) and muscle (Sinha et al. 2014); however, its effects on muscle remain under discussion (Egerman et al. 2015; Hinken et al. 2016). More work is warranted to further identify systemic rejuvenating factors that can promote neurogenesis in the aged brain.

In addition to identifying pro-youthful factors in young blood that might boost neurogenesis, an emerging body of work has identified aged blood plasma, and the factors contained therein, as a potent driver of brain aging. Consistent with the effects observed in heterochronic parabiosis and catheter-based blood exchange studies, blood plasma isolated from aged mice exerts anti-neurogenic effects on hippocampal neurogenesis when administered by I.V. injection in young mice (Villeda et al. 2011). Interestingly, old serum inhibits NSC function, in vitro, leading to decreased selfrenewal (Villeda et al. 2011) and NPC proliferation
(Bickford et al. 2015) in rodent models. Together, these data indicate the existence of soluble pro-aging factors that inhibit neurogenesis.

Multianalyte profiling of blood plasma from young and old mice, as well as from young isochronic and heterochronic parabionts, has identified six factors (B2M, CCL11, CCL12, CCL19, CCL2, and Haptoglobin) that are elevated in young heterochronic parabionts, and whose blood concentrations inversely correlate with the age-related decline in neurogenesis (Villeda et al. 2011). Of these factors, B2M, a non-covalently bound subunit of MHC class 1, and the chemokines CCL11 and CCL2 have been shown to be elevated in the blood of aged healthy humans (Targowski et al. 2005; Shurin et al. 2007; Mátrai et al. 2009; Villeda et al. 2011; Smith et al. 2015; Valiathan et al. 2016). Independent of their canonical immune functions, B2M, CCL11, and CCL2 have all been implicated as potential negative regulators of adult hippocampal neurogenesis (Villeda et al. 2011; Lee et al. 2013; Smith et al. 2015). For example, while it is yet unclear if the systemic increase of CCL2 with age contributes to the decline in adult neurogenesis, in the context of recovery from irradiation, CCL2 knockout mice exhibited increased neurogenesis after cranial irradiation (Lee et al. 2013). To date, mimicking an aged systemic environment by increasing either B2M or CCL11 has been shown to impair hippocampal neurogenesis in young mice (Villeda et al. 2011; Smith et al. 2015). In particular, systemic administration of B2M resulted in a decrease in the number of NPCs, immature neurons, and newly formed mature neurons in the hippocampi of young mice (Smith et al. 2015). Similarly, young mice given systemic injections of CCL11 had decreased adult neurogenesis compared to vehicle-treated controls (Villeda et al. 2011). Both B2M and CCL11 also inhibit neurogenesis when locally administered by stereotaxic injection into the DG of the hippocampus, and administration of B2M or CCL11 in vitro also inhibits NPC function (Villeda et al. 2011; Smith et al. 2015). Together, these studies suggest that these factors are able to directly regulate NSC function. Supporting this possibility, CCL11 readily crosses the blood-brain barrier (BBB) (Erickson et al. 2014). Additionally, while it is unknown whether B2M crosses the BBB, exposure to old blood through parabiosis or catheter-based blood exchange leads to elevated levels of B2M in the hippocampi of young mice (Smith et al. 2015; Rebo et al. 2016).

In addition to the factors identified through parabiosis, a number of other potential pro-aging factors have been identified, such as TGF- $\beta$, IL- 6 , and TNF- $\alpha$. Indeed, TGF- $\beta$ levels in blood are elevated in aged mice and systemic attenuation of TGF- $\beta$ by pharmacological inhibition of its receptor, Alk5, resulted in increased hippocampal neurogenesis in aged mice that had also received a muscle injury (Yousef et al. 2015b). While administration of TGF- $\beta 1$ to cultured NPCs has been shown to inhibit proliferation (Buckwalter et al. 2006; Yousef 
et al. 2015b) suggesting a direct regulatory effect of TGF- $\beta$ signaling on neurogenesis, inhibition of TGF- $\beta$ signaling in vivo was accompanied by a decrease in hippocampal B2M expression (Yousef et al. 2015b), suggesting that TGF- $\beta$ may influence neurogenesis through multiple mechanisms. Additionally, aging is accompanied by increased IL-6 and TNF- $\alpha$ in the blood of humans (Bruunsgaard 2006; Valiathan et al. 2016). As both these factors have been shown to inhibit murine NPC function in vitro, (Ben-Hur et al. 2003; Monje et al. 2003), it is possible that they further contribute to the age-related decline in neurogenesis. Whether preventing the age-related accumulation of these pro-aging factors can boost neurogenesis in the aged brain remains to be determined.

Based on these studies, a model has emerged in which mammalian aging drives a decline in pro-youthful factors and concomitant accumulation of detrimental pro-aging immune factors in blood that direct the age-related decline in neurogenesis (Fig. 1) (Bouchard and Villeda 2015). While in vitro studies suggest that pro-aging factors can directly affect NPC function, more research is warranted to determine the mechanisms by which pro-aging factors exert their antineurogenic affects. Furthermore, the underlying cellular sources responsible for the accumulation of pro-aging factors in old blood remain unknown. However, given their immune origin, it is possible that accumulation of pro-aging factors reflects a pro-inflammatory remodeling of the peripheral immune system.

\section{Immune cells: loss of neurotrophic and gain of anti-neurogenic function}

Aging drives many functional and structural changes in the hematopoietic system, resulting in impaired immune function (immunosenescence), increased rates of anemia, and increased incidence of myeloid malignancies (Wahlestedt et al.

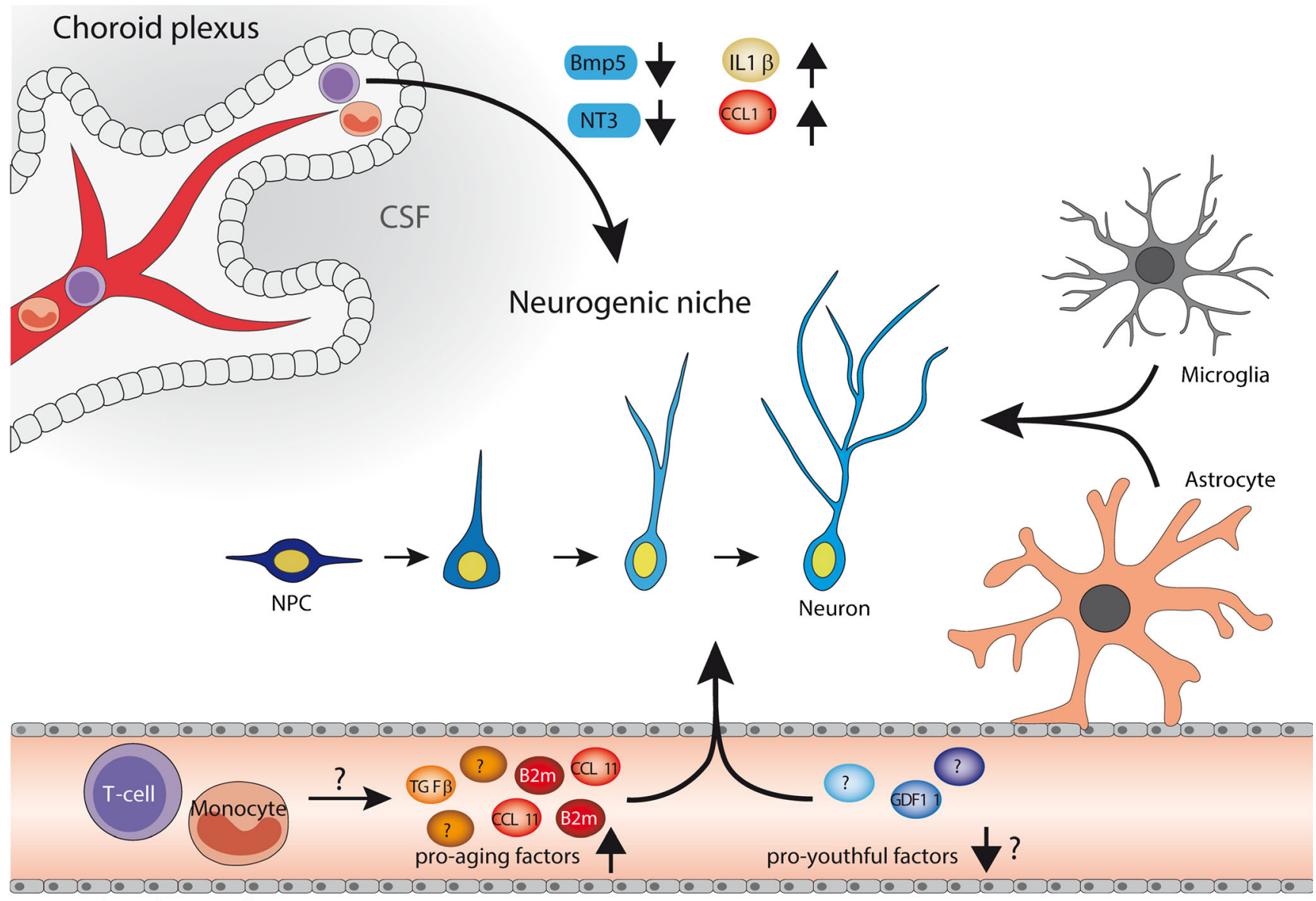

Blood vessel

Fig. 1 Potential mechanisms by which the aging systemic environment regulates the age-related decline in neurogenesis in the adult brain. Schematic illustration highlighting possible mechanisms by which agerelated changes in blood may regulate neurogenesis in the aged mammalian brain. Age-related changes in immune cells ( $\mathrm{T}$ cells and monocytes) and soluble factors in aging blood may regulate neurogenesis directly by modulating neural stem/progenitor cell function, or indirectly by altering signaling in the local neurogenic niche and choroid plexus. Arrows denote potential pathways by which blood aging affect neurogenesis. Mechanisms yet to be determined are denoted by a question mark (?). $N P C$ neural progenitor cell; $C S F$ cerebral spinal fluid; $B 2 m$ B2-microglobulin; $T G F \beta$ transforming growth factor; $C C L 11$ C-C motif chemokine $11 ; N T 3$ neurotrophin $3 ; B M P 5$ bone morphogenetic protein $5 ; I L-1 \beta$ interleukin $1 \beta$ 
2015). Specifically, aging results in decreased production of naïve $\mathrm{T}$ cells, and peripheral expansion of senescent memory and effector T cells (Dorshkind et al. 2009) that may promote an inflammatory systemic milieu as a result of their elevated cytokine production (Effros 2007; Huang et al. 2008; Montecino-Rodriguez et al. 2013). Similarly, there is reduced production of B cells, and an accumulation of memory cells with increased production of autoreactive antibodies (Dorshkind et al. 2009). Accompanying these changes to the adaptive immune system is the progressive dysregulation of the innate immune system, which includes the functional decline of neutrophils, natural killer cells, monocytes/macrophages, and dendritic cells (Shaw et al. 2010). Together, these changes are poised to contribute to the accumulation of proaging factors in old blood, and thereby inhibit neurogenesis (Fig. 1). Indeed, increased transcription of two potential proaging factors, B2M and IL-6, have been reported in aged human peripheral blood mononuclear cells (PBMCs) (Snyder-Mackler et al. 2014).

In addition to the possibility that pro-inflammatory changes to the immune system leads to increased levels of pro-aging factors, it is possible that the aging hematopoietic system also loses neurotrophic properties with age (Fig. 1). This idea has gained traction given the emerging data demonstrating a proneurogenic role of peripheral blood immune cells in young adult mice. For example, mice lacking either Ly6C(hi) monocytes or $\mathrm{T}$ cells have impaired neurogenesis, and adoptive transfers of CD4+, but not CD8+, T cells, into T celldeficient mice enhances neurogenesis (Ziv et al. 2006; Wolf et al. 2009; Möhle et al. 2016). Given that monocyte populations and $\mathrm{T}$ cells have both been shown to exhibit functional decline during age (Dorshkind et al. 2009; Shaw et al. 2010), it is possible that cellular aging of the immune system results in a loss of neurotrophic function during age (Fig. 1). Thus, it has been hypothesized that rejuvenating the immune system could counter brain aging and promote adult neurogenesis (RonHarel and Schwartz 2009). Supporting this hypothesis, it has been demonstrated that a single intravenous injection of human umbilical cord blood mononuclear cells (hUCBMC) can increase proliferation and neuronal differentiation in the DG of the hippocampi in aged but not young rats (Bachstetter et al. 2008). Interestingly, in similar experiments, administration of adult PBMCs had no effect on neurogenesis, indicating that the young age of the mononuclear cells is critical for its proneurogenic effects (Bachstetter et al. 2008). Furthermore, consistent with previous work suggesting a neurotrophic function of CD4+ T cells (Ziv et al. 2006; Wolf et al. 2009), the rejuvenating effects of hUCBC transplantation were later recapitulated by a single dose of human umbilical cord blood (hUCB)-derived CD4+ T cells, and not by administration of other types of mononuclear cells (Shahaduzzaman et al. 2013). At the cellular level, cultured rat NSCs grown in hUCB-derived $\mathrm{T}$ cell-conditioned media exhibited increased proliferation and viability compared with conditioned media from other hUCBMCs (Shahaduzzaman et al. 2013), suggesting that the effect could be mediated by secreted factors from the young T cells. While the mechanism by which hUCB cells promote neurogenesis remains to be elucidated, given that peripheral blood $\mathrm{T}$ cells and monocytes are rarely found in the brain parenchyma of healthy mice, it is likely that the effects of the immune system on neurogenesis in the aged brain are mediated through indirect mechanisms affecting the blood-brain interface.

\section{Choroid plexus: reflections of blood aging at the blood-brain interface}

The choroid plexus (CP) is a highly vascular tissue located in the ventricles of the mammalian brain that produces CSF and acts as an interface between the periphery and the brain. Secreted factors from the CP bathe the SVZ neurogenic niche and are thought to influence neurogenesis in development and adulthood (Lehtinen et al. 2013). Moreover, SVZ NSCs contact CSF directly, via apical processes (Fuentealba et al. 2012), highlighting their vulnerability to changes in the CSF. Aging drives an inflammatory transcriptional profile in the $\mathrm{CP}$ (Baruch et al. 2014; Silva-Vargas et al. 2016), and has been associated with an upregulation of type I interferon (IFN-I) dependent gene expression and decreased IFN-II expression (Baruch et al. 2014). Furthermore, aging results in an altered secretome by the $\mathrm{CP}$, which may result in decreased neurotrophic capacity. Recently, it has been shown that aging CPconditioned media contains decreased levels of BMP5 and IGF-1 that normally promote neurogenesis in the SVZ (Silva-Vargas et al. 2016). The aged CP also exhibits elevated expression of IL-6 and CCL11 (Baruch et al. 2013), both of which have been shown to inhibit neurogenesis (Monje et al. 2003; Villeda et al. 2011). Additionally, CCL11 and B2M levels are also elevated in CSF from aged humans (Villeda et al. 2011; Smith et al. 2015). Together, these studies support a model in which age-related changes in the $\mathrm{CP}$ lead to changes in the composition of soluble factors in the CSF, which contributes to the age-related decline in adult neurogenesis.

Recent evidence now suggests a direct functional role for $\mathrm{CP}$ aging and age-related decline in neurogenesis in the brain. For example, conditioned media from aged $\mathrm{CP}$ explants inhibits adult SVZ neurogenesis compared to young CPconditioned media when infused into the lateral ventricles of young mice in vivo, or administered on young SVZ NSCs in vitro (Silva-Vargas et al. 2016). Conversely, in aged mice, young $\mathrm{CP}$-conditioned media rejuvenates adult SVZ neurogenesis in vivo, and enhances self-renewal of aged SVZ NPCs in vitro (Silva-Vargas et al. 2016). Interestingly, activated NSCs and not later progenitor populations appear to be highly sensitive to age-related changes in $\mathrm{CP}$-conditioned 
media, as heterochronic infusions of $\mathrm{CP}$-conditioned media affected the numbers of proliferating SVZ NSCs in vivo, but not numbers of proliferating progenitors (Silva-Vargas et al. 2016). Furthermore, in vitro, purified activated NSCs were highly sensitive to the effects of heterochronic CPconditioned media on colony formation while transitamplifying cells and quiescent NSCs were not (Silva-Vargas et al. 2016). In addition to affecting SVZ neurogenesis, CP aging also influences hippocampal neurogenesis. Inhibition of the age-related IFN-I expression profile, through administration of an IFN-I receptor neutralizing antibody to the CSF of aged mice, increased the number of proliferating cells and immature neurons in the DG of aged mice (Baruch et al. 2014).

Given its highly vascular nature, it is likely that the CP may be sensitive to changes in soluble pro-aging factors in blood. Additionally, CD4+ T cells have recently been observed in the CP of healthy young and aged mice (Baruch et al. 2013), suggesting a potential sensitivity to cellular changes in the aged immune system. Supporting this idea, in the model of heterochronic parabiosis, blood age was found to modulate IFN-II expression profiles in the CP of old and young mice (Baruch et al. 2014). Interestingly, however, age-related differences in IFN-I profiles remained unchanged in parabiosis (Baruch et al. 2014), indicating that blood aging may contribute to some but not all age-related changes observed in the $\mathrm{CP}$ (Baruch et al. 2014). Of note, mice deficient for IFN-II signaling due to the absence of an IFN-y receptor exhibited decreased adult hippocampal neurogenesis (Baruch et al. 2014), suggesting that the transcriptional changes in IFN-II signaling at the CP induced by young or aged blood may be of functional consequence in mediating their effects on neurogenesis. Furthermore, young mice exposed to aged blood exhibited elevated CP-expression of the pro-aging factor, CCL11, compared to isochronic controls (Baruch et al. 2014), positing additional mechanisms by which blood aging may act through the CP to regulate adult neurogenesis. Together, these studies position the $\mathrm{CP}$ as a potential link between the systemic milieu and neurogenesis, implicating the $\mathrm{CP}$ as a regulator of the agerelated decline in neurogenesis (Fig. 1).

\section{Neurogenic niche: mediator of the aging systemic environment?}

The neurogenic niche is complex, comprised of many different cell types that influence NSC function (reviewed by Aimone et al. 2014). Vasculature, microglia, and astrocytes have all been shown support adult neurogenesis under basal conditions; however, this homeostatic relationship can be disrupted, particularly in the context of inflammation (Leiter et al. 2016). Thus, it has been postulated that agerelated changes to the neurogenic niche-which include astrogliosis, microgliosis and vasculature deterioration- may contribute to the age-related decline in adult neurogenesis (Leiter et al. 2016). Given the inflammatory remodeling that occurs in blood during aging, it is possible that the age-related changes in the neurogenic niche at least partially reflect blood aging, thereby providing an additional mechanism by which blood aging could regulates neurogenesis. In line with this, age-related changes in the systemic milieu have also been shown to regulate cellular components of the neurogenic niche. In particular, in parabioisis studies, exposure to young blood increased blood vessel volume and branching accompanied by increased neurogenesis in the SVZ of aged mice (Katsimpardi et al. 2014). Furthermore, aged mice systemically administered GDF11 exhibited increased blood vessel volume, along with increased SVZ neurogenesis (Katsimpardi et al. 2014).

In addition to modulating vasculature, age-related changes in the systemic milieu may also regulate neuroinflammation, and thereby affect neurogenesis by impairing the homeostatic capacity of microglia and astrocytes. While aging is associated with microgliosis, adoptive transfer of hUCB mononuclear cells or T cells decreased the number of activated microglia in the hippocampus of old rats, suggesting that young immune cells may dampen neuroinflammation in the aged neurogenic niche (Bachstetter et al. 2008; Shahaduzzaman et al. 2013). Conversely, given the immune origin of the pro-aging factors identified in old blood to date, it seems likely that old blood may also promote neuroinflammation and, consequently, inhibit neurogenesis. This would be consistent with heterochronic parabiosis and blood exchange studies demonstrating elevated B2M expression in the hippocampi of young mice exposed to old blood, which could reflect an increase in neuroinflammation (Smith et al. 2015; Rebo et al. 2016). Correspondingly, attenuation of TGF-B in old mice also dampens B2M expression in the hippocampi of aged mice (Yousef et al. 2015b). These data suggest that neurogenic niche may mediate the effects of heterochronic parabiosis on neurogenesis (Fig. 1); however, the full effects of aged blood on the neurogenic niche have not been fully investigated. Thus, a more thorough evaluation of the effects of blood on the neurogenic niche is warranted to determine whether and how blood aging broadly affects cellular hallmarks of brain aging.

\section{Conclusion}

A growing body of evidence has demonstrated that the systemic environment is a key mediator of age-related decline in neurogenesis. Models of heterochronic blood exchange have revealed that the age-related decline in adult neurogenesis in both the DG and SVZ can be either ameliorated or promoted by exposure to young or aged blood, respectively (Villeda 
et al. 2011; Katsimpardi et al. 2014). We propose a model in which blood aging results in both a loss-of-neurotrophicfunction and a concomitant gain-of-detrimental-function that negatively regulate NSC function with age (Fig. 1). At the molecular level, old blood plasma - and in particular soluble immune factors elevated therein, such as B2M and CCL11inhibit adult neurogenesis (Villeda et al. 2011; Smith et al. 2015). At the cellular level, increasing evidence indicates that peripheral blood immune cells regulate neurogenesis in adult mice (Ziv et al. 2006; Wolf et al. 2009; Möhle et al. 2016), which may be disrupted by age-related immunosenescence. Furthermore, the CP, which serves as an interface between the brain and blood, has now been proposed as a potential mediator of these immune-related effects. Indeed, exposure to young and old blood modulates transcriptional hallmarks of CP aging (Baruch et al. 2014) that may in turn affect the CP secretome, shown to modulate neurogenesis in an agedependent manner. At the neurogenic niche level, aging results in altered vasculature, microgliosis and astrogliosis, all of which can in turn regulate neurogenesis (Leiter et al. 2016). While exposure to young blood has been shown to increase vasculature in the aged mouse SVZ (Katsimpardi et al. 2014), the extent to which the aging systemic environment alters the neurogenic niche is not fully understood. Ultimately, while the systemic environment has now been identified at the interface of aging and adult neurogenesis, much work remains to fully elucidate the intricate and multifaceted mechanisms by which the systemic environment promotes loss of neurogenic capacity in the aged mammalian brain. Additionally, an emerging field of aging research is now looking to models of extended lifespan and delayed aging to explore the potential for rejuvenation. It will be of interest to examine the link between the aging systemic environment and neurogenesis in models in which aging process is protracted, such as the naked mole rat (Penz et al. 2015), in order to gain insights into protective mechanisms that delay the aging process.

Acknowledments We thank Gregor Bieri for graphical illustrations. This work was supported by the ARCS foundation (L.K.S.), National Institutes of Health Director's Independence Award (DP5-OD012178, S.A.V), and National Institute on Aging (R01-AG053382, R01AG055797, S.A.V).

\section{Compliance with ethical standards}

Competing interests statement The authors declare that they have no competing financial interests.

Open Access This article is distributed under the terms of the Creative Commons Attribution 4.0 International License (http:// creativecommons.org/licenses/by/4.0/), which permits unrestricted use, distribution, and reproduction in any medium, provided you give appropriate credit to the original author(s) and the source, provide a link to the Creative Commons license, and indicate if changes were made.

\section{References}

Aimone JB, Li Y, Lee SW et al (2014) Regulation and function of adult neurogenesis: from genes to cognition. Physiol Rev 94:991-1026. https://doi.org/10.1152/physrev.00004.2014

Altman J, Das GD (1965) Autoradiographic and histological evidence of postnatal hippocampal neurogenesis in rats. J Comp Neurol 124: 319-335. https://doi.org/10.1002/cne.901240303

Amrein I, Isler K, Lipp HP (2011) Comparing adult hippocampal neurogenesis in mammalian species and orders: influence of chronological age and life history stage. Eur J Neurosci 34:978-987. https://doi.org/10.1111/j.1460-9568.2011.07804.x

Bachstetter AD, Pabon MM, Cole MJ et al (2008) Peripheral injection of human umbilical cord blood stimulates neurogenesis in the aged rat brain. BMC Neurosci 9:22. https://doi.org/10.1186/1471-2202-9-22

Baht GS, Silkstone D, Vi L et al (2015) Exposure to a youthful circulaton rejuvenates bone repair through modulation of $\beta$-catenin. Nat Commun 6:7131. https://doi.org/10.1038/ncomms8131

Barker JM, Boonstra R, Wojtowicz JM (2011) From pattern to purpose: how comparative studies contribute to understanding the function of adult neurogenesis. Eur J Neurosci 34:963-977. https://doi.org/10. 1111/j.1460-9568.2011.07823.x

Baruch K, Deczkowska A, David E et al (2014) Aging-induced type I interferon response at the choroid plexus negatively affects brain function. Science 346:89-93. https://doi.org/10.1126/science. 1252945

Baruch K, Ron-Harel N, Gal H et al (2013) CNS-specific immunity at the choroid plexus shifts toward destructive Th2 inflammation in brain aging. Proc Natl Acad Sci U S A 110:2264-2269. https://doi.org/10. 1073/pnas. 1211270110

Ben-Hur T, Ben-Menachem O, Furer V et al (2003) Effects of proinflammatory cytokines on the growth, fate, and motility of multipotential neural precursor cells. Mol Cell Neurosci 24:623-631. https://doi. org/10.1016/S1044-7431(03)00218-5

Bickford PC, Kaneko Y, Grimmig B et al (2015) Nutraceutical intervention reverses the negative effects of blood from aged rats on stem cells. Age (Omaha). https://doi.org/10.1007/s11357-015-9840-7

Bizon JL, Lee HJ, Gallagher M (2004) Neurogenesis in a rat model of age-related cognitive decline. Aging Cell 3:227-234. https://doi.org/ 10.1111/j.1474-9728.2004.00099.x

Bond AM, Ming G-L, Song H (2015) Adult mammalian neural stem cells and neurogenesis: five decades later. Cell Stem Cell 17:385-395. https://doi.org/10.1016/j.stem.2015.09.003

Bouchard J, Villeda SA (2015) Aging and brain rejuvenation as systemic events. J Neurochem 132:5-19

Brack AS, Conboy MJ, Roy S et al (2007) Increased Wnt signaling during aging alters muscle stem cell fate and increases fibrosis. Science 317:807-810. https://doi.org/10.1126/science.1144090

Bruunsgaard H (2006) The clinical impact of systemic low-level inflammation in elderly populations. With special reference to cardiovascular disease, dementia and mortality. Dan Med Bull 53:285-309

Buckwalter MS, Yamane M, Coleman BS et al (2006) Chronically increased transforming growth factor-betal strongly inhibits hippocampal neurogenesis in aged mice. Am J Pathol 169:154-164. https://doi.org/10.2353/ajpath.2006.051272

Castellano JM, Kirby ED, Wyss-Coray T (2015) Blood-borne revitalization of the aged brain. JAMA Neurol 72:1191-1194. https://doi.org/ 10.1001/jamaneurol.2015.1616

Castellano JM, Mosher KI, Abbey RJ et al (2017) Human umbilical cord plasma proteins revitalize hippocampal function in aged mice. Nature 544:488-492. https://oi.org/10.1038/nature22067

Chapouton P, Jagasia R, Bally-Cuif L (2007) Adult neurogenesis in nonmammalian vertebrates. BioEssays 29:745-757 
Christian KM, Song H, Ming G (2014) Functions and dysfunctions of adult hippocampal neurogenesis. Annu Rev Neurosci 37:243-262. https://doi.org/10.1146/annurev-neuro-071013-014134

Clelland CD, Choi M, Romberg C et al (2009) A functional role for adult hippocampal neurogenesis in spatial pattern separation. Science 325:210-213. https://doi.org/10.1126/science.1173215

Conboy IM, Conboy MJ, Wagers AJ et al (2005) Rejuvenation of aged progenitor cells by exposure to a young systemic environment. Nature 433:760-764. https://doi.org/10.1038/nature03260

Deng W, Saxe MD, Gallina IS, Gage FH (2009) Adult-born hippocampal dentate granule cells undergoing maturation modulate learning and memory in the brain. J Neurosci 29:13532-13542. https://doi.org/ 10.1523/JNEUROSCI.3362-09.2009

Dorshkind K, Montecino-Rodriguez E, Signer RAJ (2009) The ageing immune system: is it ever too old to become young again? Nat Rev Immunol 9:57-62. https://doi.org/10.1038/nri2471

Drapeau E, Mayo W, Aurousseau C et al (2003) Spatial memory performances of aged rats in the water maze predict levels of hippocampal neurogenesis. Proc Natl Acad Sci U S A 100:14385-14390. https:// doi.org/10.1073/pnas.2334169100

Effros RB (2007) Role of T lymphocyte replicative senescence in vaccine efficacy. Vaccine 25:599-604

Egerman MA, Cadena SM, Gilbert JA et al (2015) GDF11 increases with age and inhibits skeletal muscle regeneration. Cell Metab 22:164 174. https://doi.org/10.1016/j.cmet.2015.05.010

Enwere E (2004) Aging results in reduced epidermal growth factor receptor signaling, diminished olfactory neurogenesis, and deficits in fine olfactory discrimination. J Neurosci 24:8354-8365. https://doi. org/10.1523/JNEUROSCI.2751-04.2004

Erickson MA, Morofuji Y, Owen JB, Banks WA (2014) Rapid transport of CCL11 across the blood-brain barrier: regional variation and importance of blood cells. J Pharmacol Exp Ther 349:497-507. https:// doi.org/10.1124/jpet.114.213074

Fuentealba LC, Obernier K, Alvarez-Buylla A (2012) Adult neural stem cells bridge their niche. Cell Stem Cell 10:698-708. https://doi.org/ 10.1016/j.stem.2012.05.012

Hinken AC, Powers JM, Luo G et al (2016) Lack of evidence for GDF11 as a rejuvenator of aged skeletal muscle satellite cells. Aging Cell 15:582-584. https://doi.org/10.1111/acel.12475

Huang M-C, Liao J-J, Bonasera S et al (2008) Nuclear factor-kappaBdependent reversal of aging-induced alterations in T cell cytokines. FASEB J 22:2142-2150. https://doi.org/10.1096/fj.07-103721

Katsimpardi L, Litterman NK, Schein PA et al (2014) Vascular and neurogenic rejuvenation of the aging mouse brain by young systemic factors. Science 344:630-634. https://doi.org/10.1126/science. 1251141

Kempermann G, Gast D, Gage FH (2002) Neuroplasticity in old age: sustained fivefold induction of hippocampal neurogenesis by longterm environmental enrichment. Ann Neurol 52:135-143. https:// doi.org/10.1002/ana.10262

Kodali M, Parihar VK, Hattiangady B et al (2015) Resveratrol prevents age-related memory and mood dysfunction with increased hippocampal neurogenesis and microvasculature, and reduced glial activation. Sci Rep 5:8075. https://doi.org/10.1038/srep08075

Lee SW, Haditsch U, Cord BJ et al (2013) Absence of CCL2 is sufficient to restore hippocampal neurogenesis following cranial irradiation. Brain Behav Immun 30:33-44

Lehtinen MK, Bjornsson CS, Dymecki SM et al (2013) The choroid plexus and cerebrospinal fluid: emerging roles in development, disease, and therapy. J Neurosci 33:17553-17559. https://doi.org/10. 1523/JNEUROSCI.3258-13.2013

Leiter O, Kempermann G, Walker TL (2016) A common language: how neuroimmunological cross talk regulates adult hippocampal neurogenesis. Stem Cells Int 2016:1681590. https://doi.org/10. $1155 / 2016 / 1681590$
Loffredo FS, Steinhauser ML, Jay SM et al (2013) Growth differentiation factor 11 is a circulating factor that reverses age-related cardiac hypertrophy. Cell 153:828-839. https://doi.org/10.1016/j.cell.2013. 04.015

Lugert S, Basak O, Knuckles P et al (2010) Quiescent and active hippocampal neural stem cells with distinct morphologies respond selectively to physiological and pathological stimuli and aging. Cell Stem Cell 6:445-456. https://doi.org/10.1016/j.stem.2010.03.017

Mátrai Z, Németh J, Miklós K et al (2009) Serum beta2-microglobulin measured by immunonephelometry: expression patterns and reference intervals in healthy adults. Clin Chem Lab Med 47:585-589. https://doi.org/10.1515/CCLM.2009.137

Merrill DA, Karim R, Darraq M et al (2003) Hippocampal cell genesis does not correlate with spatial learning ability in aged rats. J Comp Neurol 459:201-207. https://doi.org/10.1002/cne.10616

Möhle L, Mattei D, Heimesaat MM et al (2016) Ly6Chi monocytes provide a link between antibiotic-induced changes in gut microbiota and adult hippocampal neurogenesis. Cell Rep 15:1945-1956. https://doi.org/10.1016/j.celrep.2016.04.074

Molofsky AV, Slutsky SG, Joseph NM et al (2006) Increasing p16INK4a expression decreases forebrain progenitors and neurogenesis during ageing. Nature 443:448-452. https://doi.org/10.1038/nature05091

Monje ML, Toda H, Palmer TD (2003) Inflammatory blockade restores adult hippocampal neurogenesis. Science 302:1760-1765. https:// doi.org/10.1126/science.1088417

Montecino-Rodriguez E, Berent-Maoz B, Dorshkind K (2013) Causes, consequences, and reversal of immune system aging. J Clin Invest 123:958-965. https://doi.org/10.1172/JCI16096

Nottebohm F (2004) The road we travelled: discovery, choreography, and significance of brain replaceable neurons. Ann N Y Acad Sci 1016: 628-658. https://doi.org/10.1196/annals.1298.027

Penz OK, Fuzik J, Kurek AB et al (2015) Protracted brain development in a rodent model of extreme longevity. Sci Rep 5:11592. https://doi. org $/ 10.1038 /$ srep 11592

Rebo J, Mehdipour M, Gathwala R et al (2016) A single heterochronic blood exchange reveals rapid inhibition of multiple tissues by old blood. Nat Commun 7:13363. https://doi.org/10.1038/ ncomms 13363

Ron-Harel N, Schwartz M (2009) Immune senescence and brain aging: can rejuvenation of immunity reverse memory loss? Trends Neurosci 32:367-375. https://doi.org/10.1016/j.tins.2009.03.003

Ruckh JM, Zhao JW, Shadrach JL et al (2012) Rejuvenation of regeneration in the aging central nervous system. Cell Stem Cell 10:96-103. https://doi.org/10.1016/j.stem.2011.11.019

Sahay A, Wilson DA, Hen R (2011) Pattern separation: a common function for new neurons in hippocampus and olfactory bulb. Neuron 70: $582-588$

Salpeter SJ, Khalaileh A, Weinberg-Corem N et al (2013) Systemic regulation of the age-related decline of pancreatic $\beta$-cell replication. Diabetes 62:2843-2848. https://doi.org/10.2337/db13-0160

Seib DRM, Corsini NS, Ellwanger K et al (2013) Loss of dickkopf-1 restores neurogenesis in old age and counteracts cognitive decline. Cell Stem Cell 12:204-214. https://doi.org/10.1016/j.stem.2012.11. 010

Shahaduzzaman M, Golden JE, Green S et al (2013) A single administration of human umbilical cord blood $\mathrm{T}$ cells produces long-lasting effects in the aging hippocampus. Age (Omaha) 35:2071-2087. https://doi.org/10.1007/s11357-012-9496-5

Shaw AC, Joshi S, Greenwood H et al (2010) Aging of the innate immune system. Curr Opin Immunol 22:507-513

Shetty AK, Hattiangady B, Shetty GA (2005) Stem/progenitor cell proliferation factors FGF-2, IGF-1, and VEGF exhibit early decline during the course of aging in the hippocampus: role of astrocytes. Glia 51:173-186. https://doi.org/10.1002/glia.20187 
Shurin GV, Yurkovetsky ZR, Chatta GS et al (2007) Dynamic alteration of soluble serum biomarkers in healthy aging. Cytokine 39:123129. https://doi.org/10.1016/j.cyto.2007.06.006

Silva-Vargas V, Maldonado-Soto AR, Mizrak D et al (2016) Agedependent niche signals from the choroid plexus regulate adult neural stem cells. Cell Stem Cell 19:643-652. https://doi.org/10.1016/j. stem.2016.06.013

Sinha M, Jang YC, Oh J et al (2014) Restoring systemic GDF11 levels reverses age-related dysfunction in mouse skeletal muscle. Science 344:649-652. https://doi.org/10.1126/science.1251152

Smith LK, He Y, Park J-S et al (2015) $\beta 2$-microglobulin is a systemic pro-aging factor that impairs cognitive function and neurogenesis. Nat Med 21:932-937. https://doi.org/10.1038/nm.3898

Snyder-Mackler N, Somel M, Tung J (2014) Shared signatures of social stress and aging in peripheral blood mononuclear cell gene expression profiles. Aging Cell 13:954-957. https://doi.org/10.1111/acel. 12239

Targowski T, Jahnz-Rózyk K, Plusa T, Glodzinska-Wyszogrodzka E (2005) Influence of age and gender on serum eotaxin concentration in healthy and allergic people. J Investig Allergol Clin Immunol 15: 277-282

Tsai C-Y, Tsai C-Y, Arnold SJ, Huang G-J (2015) Ablation of hippocampal neurogenesis in mice impairs the response to stress during the dark cycle. Nat Commun 6:8373. https://doi.org/10.1038/ ncomms 9373

Valiathan R, Ashman M, Asthana D (2016) Effects of ageing on the immune system: infants to elderly. Scand J Immunol 83:255-266. https://doi.org/10.1111/sji.12413

Valley MT, Mullen TR, Schultz LC et al (2009) Ablation of mouse adult neurogenesis alters olfactory bulb structure and olfactory fear conditioning. Front Neurosci. https://doi.org/10.3389/neuro.22.003. 2009

van Praag H, Shubert T, Zhao C, Gage FH (2005) Exercise enhances learning and hippocampal neurogenesis in aged mice. J Neurosci 25:8680-8685. https://doi.org/10.1523/JNEUROSCI.1731-05.2005
Villeda SA, Luo J, Mosher KI et al (2011) The ageing systemic milieu negatively regulates neurogenesis and cognitive function. Nature 477:90-94. https://doi.org/10.1038/nature10357

Villeda SA, Plambeck KE, Middeldorp J et al (2014) Young blood reverses age-related impairments in cognitive function and synaptic plasticity in mice. Nat Med 20:659-663. https://doi.org/10.1038/ nm.3569

Wahlestedt M, Pronk CJ, Bryder D (2015) Concise review: hematopoietic stem cell aging and the prospects for rejuvenation. Stem Cells Transl Med 4:186-194. https://doi.org/10.5966/sctm.2014-0132

Wolf SA, Steiner B, Akpinarli A et al (2009) CD4-positive T lymphocytes provide a neuroimmunological link in the control of adult hippocampal neurogenesis. J Immunol 182:3979-3984. https://doi. org/10.4049/jimmunol.0801218

Yousef H, Morgenthaler A, Schlesinger C et al (2015a) Age-associated increase in BMP signaling inhibits hippocampal neurogenesis. Stem Cells 33:1577-1588. https://doi.org/10.1002/stem.1943

Yousef H, Conboy MJ, Morgenthaler A (2015b) Systemic attenuation of the TGF- $\beta$ pathway by a single drug simultaneously rejuvenates hippocampal neurogenesis and myogenesis in the same old mammal. Neurobiol Dis 74:1-8. https://doi.org/10.1016/j.nbd.2014.11. 016

Zhang C-L, Zou Y, He W et al (2008) A role for adult TLX-positive neural stem cells in learning and behaviour. Nature 451:1004 1007. https://doi.org/10.1038/nature06562

Ziv Y, Ron N, Butovsky O et al (2006) Immune cells contribute to the maintenance of neurogenesis and spatial learning abilities in adulthood. Nat Neurosci 9:268-275. https://doi.org/10.1038/nn1629

Zupanc GKH, Sirbulescu RF (2013) Teleost fish as a model system to study successful regeneration of the central nervous system. Curr Top Microbiol Immunol 367:193-233. https://doi.org/10.1007/822012-297 